\title{
A Novel Energy Efficient Network Management Scheme of Heterogeneous WSN with MIMO Techniques
}

\author{
Trupti Mayee Behera \\ School of Electronics Engineering, \\ KIIT University, \\ Bhubaneswar, Odisha, India
}

\author{
Sudhansu Sekhar Singh \\ School of Electronics Engineering, \\ KIIT University, \\ Bhubaneswar, Odisha, India
}

\begin{abstract}
Deployment of sensor nodes has become a major challenge in improving network coverage dynamically in wireless sensor networks. Hence different spatial multiplexing techniques are experimented to provide energy efficiency in sensor networks. At the primary deployment level it is evident that the multiple input single outputs (MISO) consumes lesser energy than the other techniques. However implementing STBC based MIMO (SCHCT) technique in wireless sensor network further reduces the energy to large extent. This paper exploits this method for a group of sensor nodes those are divided into many clusters where multiple cluster heads cooperatively manages the transmission activity with less energy consumption and enhanced network life span. The network lifetime of WSN has also further increased by employing a different approach with the SCHCT technique even to a Heterogeneous sensor Network
\end{abstract}

\section{Keywords}

Heterogeneous wireless sensor networks, Energy efficiency, Virtual MIMO, STBC, SCHCT

\section{INTRODUCTION}

Energy management in WSN differs from normal mobile network as instead of random movement the sensor nodes of WSN should be directed purposefully. In such a case it becomes necessary to design an energy efficient network which will also ensure an effective sensor node mobilization. Hence, we start with formation of clusters which are nothing but self-organised sensor nodes, out of which one node is selected as cluster head having highest energy. As direct deployment of multiple input multiple output antennas practically in a single small sensor node is not feasible hence a virtual antenna array of nodes is formed called virtual MIMO cell where STBC can be implemented.STBC is a technique to transmit multiple copies of a data stream across a number of antennas in the form of matrix, where nodes represent the number of antennas and columns represent the time slots for data transmission

\section{SYSTEM MODEL}

Consider a WSN with say, 'N' number of sensor nodes that are randomly distributed within an area each of side say ' $M$ ' meters. Here the sensor nodes are initially assumed homogeneous, energy confined and stationary. The sink is a node acting as a base station, having unlimited available energy and one or more receiving antennas to implement the virtual MIMO communication [1]. All nodes have the capacity of sensing and transmitting the data to other nodes and sink as shown in Figure 1. The distributed sensor nodes are classified into normal sensing nodes to collect the information and some as cluster heads $(\mathrm{CH})$ which performs other important functions. The process of SCHCT, i.e. STBC based cluster heads cooperative transmission is divided into rounds [2], each round consisting of three phases.

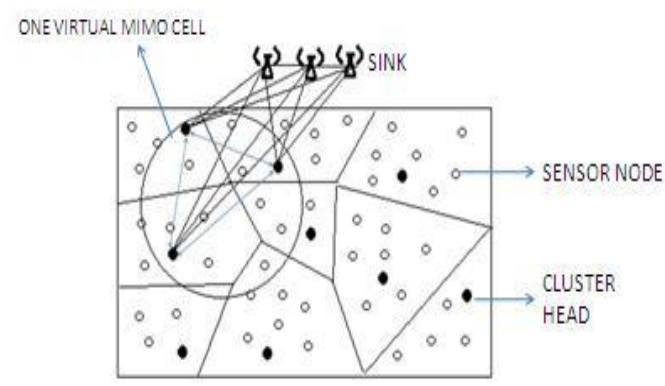

Fig1: System Model

\subsection{Cluster Formation Stage}

In this phase, all sensor nodes self-organize themselves to form clusters. Each node uses the CSMA-CA protocol to operate for the wireless channel. When a node succeeds in accessing the channel and becomes a part of the network, it sends a message carrying the node ID and discovers its one-hop neighbours [3].

The next step will be to select the cluster head. Since cluster heads consumes more energy than any normal node (used in collection, aggregation, and forwarding data), the selection of $\mathrm{CHs}$ therefore depends on the node's remaining energy. When the nodes start the clustering process, all nodes compare its remaining energy with the neighbouring nodes. As soon as a node finds the maximum energy node in its neighbourhood, it announces the node to be the $\mathrm{CH}$ and transfers the message to all neighbours.

Once the $\mathrm{CH}$ is elected, each $\mathrm{CH}$ transmits its ID using CSMA scheme to other sensor nodes in the network. And finally the cluster member sends their ID to the $\mathrm{CH}$ so that it can record it.

\subsection{Steady State Stage}

Once the clusters are formed, the $\mathrm{CH}$ sets up a TDMA schedule for the cluster nodes to transmit data. Using LEACH protocol, the cluster members transmit the collected data to their respective $\mathrm{CHs}$ in a number of frames [2]. The cluster member, in each frame transmits the sensed information in allotted time slot specified, while sleeping in rest of the slots in order to save power. Only when all $\mathrm{CHs}$ collect their respective data frames from sensor nodes, they check for any redundant bits to perform data aggregation. 


\subsection{Cooperative Transmission Stage}

The $\mathrm{CH}$ waits for a polling message after aggregating the data When it receives any broadcast message from the sink, it sends location information and the IDs of all CHs [4].On the basis of this data, the sink groups all $\mathrm{CHs}$ into a virtual MIMO antenna array and sets up a TDMA schedule. Each $\mathrm{CH}$ of the cell sends its data to other CHs in specific TDMA slots. When each of the $\mathrm{CHs}$ receives their data bits from others, the transmission sequence is decided as per STBC coding [5]. The transmission of data among the MIMO cell depends on the TDMA time slot.

\section{WORK APPROACH}

The following assumptions are made in the analysis process

i. An Additive White Gaussian Noise(AWGN) channel with squared path loss for the intra-cluster communication,

ii. A flat Rayleigh fading channel with a power loss for the inter-cluster communication

iii. BPSK will be the modulation technique.

\section{Total Power Consumed}

In order to calculate the communication energy consumed by the whole network, first the energy consumed in receiving or transmitting a single bit is modelled [6]

The energy consumed in transmitting one bit of information can be found as:

$$
\mathrm{E}_{\mathrm{bt}}=\left(\mathrm{P}_{\mathrm{PA}}+\mathrm{P}_{\mathrm{C}}\right) / \mathrm{R}_{\mathrm{b}}
$$

Where $R_{b}$ is the bit rate of the system, $P_{P A}$ is the power consumption by power amplifiers and $\mathrm{P}_{\mathrm{C}}$ is the power consumption by other circuit blocks.

If $\alpha$ is the RFPA efficiency, then the power consumption of the power amplifiers as formulated by Yuan and Chen (2006) can be stated as [7]:

$$
\mathrm{P}_{\mathrm{PA}}=(1+\alpha) \mathrm{P}_{\mathrm{ou}}
$$

Thus $\mathrm{P}_{\text {out }}$ can be estimated as:

$$
P_{\text {out }}=\left\{\frac{E_{b} R_{b} \frac{(4 \Pi)^{2} M_{l} N_{f}}{G_{t} G_{r} \lambda^{2}} d^{2} ; d \leq d_{0}}{E_{b} R_{b} \frac{M_{l} N_{f}}{G_{r} G_{t} h_{t}^{2} h_{r}^{2}} d^{4} ; d>d_{o}}\right\}
$$

In this case " $E_{b}$ is the average energy per bit at the receiver, $d$ is the transmission distance, $d_{0}$ is the threshold distance defined as $4 \pi h_{t} h_{r} / \lambda . G_{t}$ is the gain of transmitting antenna, $G_{r}$ is the gain of receiving antenna, $\lambda$ is the carrier wavelength, $h_{t}$ and $h_{r}$ are transmitting and receiving antenna heights respectively, $M_{1}$ is the link margin compensating any variations, background noise or interference. $\mathrm{N}_{\mathrm{f}}$ is the receiver noise figure".

The power consumed by receiving circuitry $\mathrm{P}_{\mathrm{cr}}$ and transmitting circuitry $\mathrm{P}_{\mathrm{ct}}$ can be written as

$$
\begin{gathered}
P_{c t} \approx P_{\text {mix }}+P_{D A C}+P_{s y n}+P_{f i l t} \\
P_{c r} \approx P_{L N A}+P_{s y n}+P_{\text {mix }}+P_{I F A}+P_{f i l}+P_{A D C}
\end{gathered}
$$

"where $\mathrm{P}_{\mathrm{DAC}}, \mathrm{P}_{\text {mix }}, \mathrm{P}_{\text {filt }}, \mathrm{P}_{\text {syn }}, \mathrm{P}_{\mathrm{LNA}}, \mathrm{P}_{\text {IFA }}, \mathrm{P}_{\text {filr }}, \mathrm{P}_{\mathrm{ADC}}$ are the power consumption for $\mathrm{D} / \mathrm{A}$ converter, the mixer, the filters at the transmitter side, the frequency synthesizer, the low noise amplifier, the intermediate frequency amplifier, the active filters at the receiver side and the A/D converter, respectively".

Hence the total power consumption for $\mathrm{N}_{\mathrm{T}}$ transmitter circuits and $\mathrm{N}_{\mathrm{R}}$ receiver circuits is:

$$
\begin{gathered}
P_{C}=N_{R}\left(P_{L N A}+P_{s y n}+P_{m i x}+P_{I F A}+P_{A D C}+P_{f i l}\right)+N_{T} \\
\left(P_{D A C}+P_{m i x}+P_{f i l t}+P_{s y n}\right)
\end{gathered}
$$

\subsection{Long haul transmission}

In the long-haul communication, the cluster nodes encode the compressed data and transmit it to the access point or sinks [8]. The energy consumed in transmitting one bit for such long distance MIMO transmission from cluster head to sink can be calculated as:

$$
\mathrm{E}_{\mathrm{bt} \_ \text {MIMO }}=(1+\alpha) \mathrm{E}_{\mathrm{b} \_ \text {MIMO }}\left\{\frac{M_{l} N_{f}}{G_{r} G_{t} h_{r}^{2} h_{t}^{2}} d_{t o S}^{4}+\frac{N_{T} P_{c t}}{R_{b}}\right\}
$$

Where $d_{t o S}$ is the distance between $\mathrm{CH}$ and sink and " $E_{b \_ \text {MIMO }}$ is the required average energy per bit for a given BER".

\subsection{Intra-cluster transmission}

The transmission comprises of sending data frames from normal nodes to the $\mathrm{CH}$ during their allocated time slot. The energy consumed in transmitting one bit from one cluster member to $\mathrm{CH}$ in a single virtual MIMO cell can be calculated as:

$$
\begin{gathered}
\mathrm{E}_{\mathrm{bt} \_ \text {intra }}=(1+\alpha) \mathrm{E}_{\mathrm{b} \_ \text {intra }} \\
\left\{\frac{(4 \Pi)^{2} M_{l} N_{f}}{G_{t} G_{r} \lambda^{2}} d_{C t o C H}^{2}+\frac{P_{c t}}{R_{b}}\right\}
\end{gathered}
$$

Where $d_{t o C H}$ is the distance between cluster members to $\mathrm{CH}$ within a single virtual MIMO cell and " $E_{b \_ \text {intra }}$ is the required energy per bit for a given BER requirement".

\subsection{Inter-cluster transmission}

The consumed energy for transmission of one bit from $\mathrm{CH}$ to $\mathrm{CH}$ in a single virtual MIMO cell can be calculated as:

$$
\mathrm{E}_{\mathrm{bt} \_ \text {inter }}=(1+\alpha) \mathrm{E}_{\mathrm{b} \_ \text {inter }}\left\{\frac{(4 \Pi)^{2} M_{l} N_{f}}{G_{t} G_{r} \lambda^{2}} d_{C t o C}^{2}+\frac{P_{c t}}{R_{b}}\right\}
$$

where $d_{C t o C}$ is the distance between $\mathrm{CHs}$ within a single virtual MIMO cell and " $E_{b_{-} i n t e r}$ is the required energy per bit for a given BER requirement".

Energy consumption of the proposed work consists of energy consumption for the cluster heads and energy consumption for the sensor nodes.

\section{Energy Consumption for cluster heads}

For $K_{c}$ number of clusters, then there can be $N / K_{c}$ nodes per cluster, so that the combined energy required in a single cluster, $\mathrm{E}_{\text {cluster }}$ can be formulated as:

$\mathrm{E}_{\text {cluster }}=\left(\mathrm{N} / \mathrm{K}_{\mathrm{c}}-1\right) \mathrm{E}_{\mathrm{s}}+\mathrm{E}_{\mathrm{CH}}$

Where $\mathrm{E}_{\mathrm{s}}$ is the energy consumed by a cluster member and $\mathrm{E}_{\mathrm{CH}}$ is the energy consumed by a cluster head.

Assuming L data bits sent by each sensor node to $\mathrm{CH}$, we can find the energy consumed by the $\mathrm{CH}$ as:

$$
\mathrm{E}_{\mathrm{CH}}=\mathrm{L}\left(\mathrm{N} / \mathrm{K}_{\mathrm{c}}-1\right) \mathrm{E}_{\mathrm{br}}+\mathrm{L}\left(\mathrm{N} / \mathrm{K}_{\mathrm{c}}\right) \mathrm{E}_{\mathrm{DA}}+\mathrm{L}\left(\mathrm{N}_{\mathrm{T}}-1\right) \mathrm{E}_{\mathrm{br}}+
$$

$$
\mathrm{LE}_{\text {bt_inter }}+\mathrm{LE}_{\text {bt_MIMO }}
$$

Where " $E_{D A}$ is the aggregation energy consumed per bit".

\section{Energy Consumption by sensor nodes}

The energy consumed by a sensor node in transmitting data is given by:

$$
\mathrm{E}_{\mathrm{s}}=\mathrm{LE}_{\mathrm{bt} \_ \text {intra }}
$$


And to receive a bit the energy consumed can be given as:

$$
\mathrm{E}_{\mathrm{br}}=\mathrm{P}_{\mathrm{cr}} /\left(\mathrm{R}_{\mathrm{b}}\right)
$$

Hence, the total energy consumed in one round of the SCHCT scheme can be derived as:

$$
\begin{aligned}
\mathrm{E}_{\text {total }} & =\mathrm{K}_{\mathrm{c}} \mathrm{E}_{\text {cluster }} \\
& =\left(\mathrm{N}-\mathrm{K}_{\mathrm{c}}\right) \mathrm{E}_{\mathrm{s}}+\mathrm{K}_{\mathrm{c}} \mathrm{E}_{\mathrm{CH}}
\end{aligned}
$$

The above equation gives the total energy consumption in an $\mathrm{M} \times \mathrm{M}$ MIMO network. By approximating the bound as equality, we can calculate the total energy consumption per bit for the MISO system as:

$E_{\text {MISO }}=(1+\alpha)\left[N_{t} N_{o} / P_{b}{ }^{1 / M t}\right] \times\left[(4 \pi d)^{2} / G_{t} G_{r} \lambda^{2}\right] \times\left[M_{l} N_{f}\right]+$ $\left(P_{c} / R_{b}\right)$

\section{HETEROGENEOUS WSN}

Initially we had assumed the WSN with homogenous nodes, but now we consider the case where a percentage of the total sensor nodes drive more energy resources than the rest of the nodes and all distributed uniformly. Let ' $m$ ' be the number of nodes equipped with ' $\alpha$ ' times more energy than other normal sensors. These powerful nodes can be termed as advanced nodes, and the rest remain normal nodes [9].

The probability of a normal node getting elected as $\mathrm{CH}$ is $\mathrm{P}_{\text {normal }}$ given by:

$$
\mathrm{P}_{\text {normal }}=\frac{P}{1+\alpha \times m}
$$

$\mathrm{T}(\mathrm{i})=\left\{\frac{\text { Pnormal }}{1-\text { Pnormal } \times\left(r \bmod \frac{1}{\text { Pnormal }}\right)}\right.$

if $i \in \mathrm{G}_{\text {normal }}, 0$ otherwise

Probability of an advanced node getting elected as cluster-head $\mathrm{P}_{\text {advanced }}$ given as

$$
\mathrm{P}_{\text {advanced }}=\frac{P}{1+\alpha \times m}(1+\alpha)
$$

$$
\mathrm{T}(\mathrm{i})=\frac{\text { Padvanced }}{1-\text { Padvanced } \times r \bmod \frac{1}{\text { Padvanced }}}
$$

if $i \in \mathrm{G}_{\text {advanced }}, 0$ otherwise

Using SCHCT in collaboration with heterogeneity, we can infer the first node to die in a round will be nearer to the sink which is also the case if we consider homogeneous distribution. Also, it is sure that the first node to die is a normal node. It is obvious that in the next rounds the chances of an advanced node to die is much less as compared a normal node. Hence as the number of normal nodes decreases there comes a time when only advanced nodes would remain alive and contribute in enhancing the network lifetime.

Assume a heterogeneous sensor network of $100 \times 100 \mathrm{~m}^{2}$. We symbolize ' $\times$ ' as the sink,' $*$ ' as a cluster head, ' $O$ ' as a normal node, ' + ' as an advanced node, and ' $\bullet$ ' for a dead node,

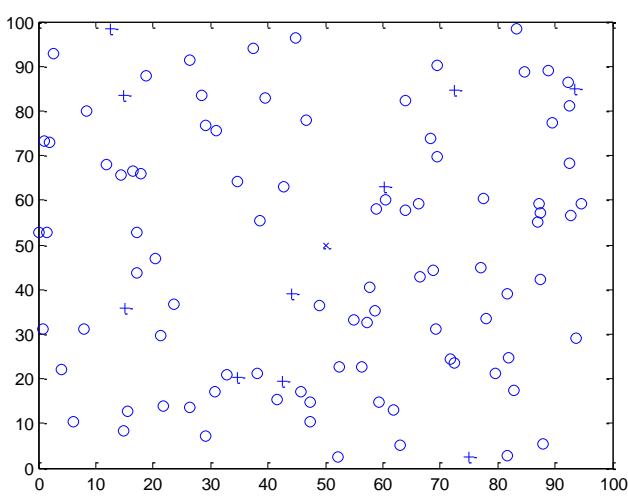

Figure 2: WSN with advanced and normal nodes

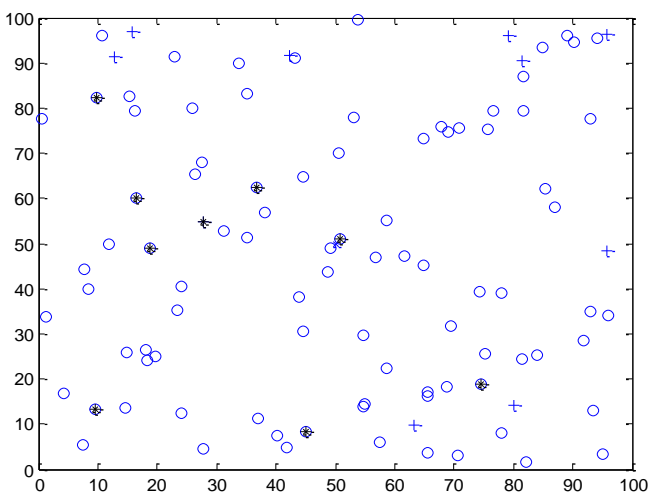

Figure 3: All of the nodes are alive

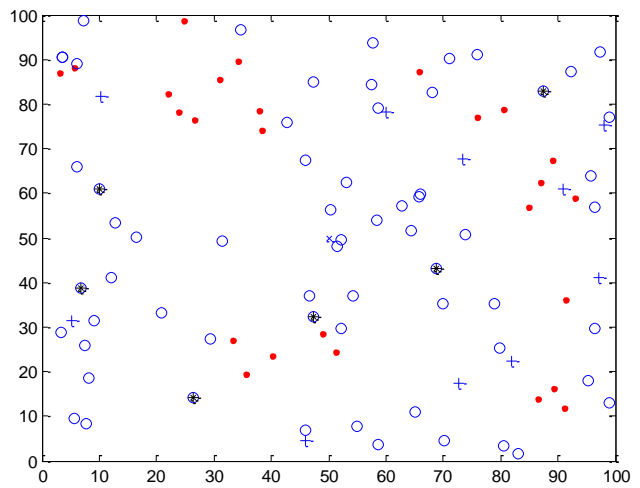

Figure 4: Some of the nodes are dead

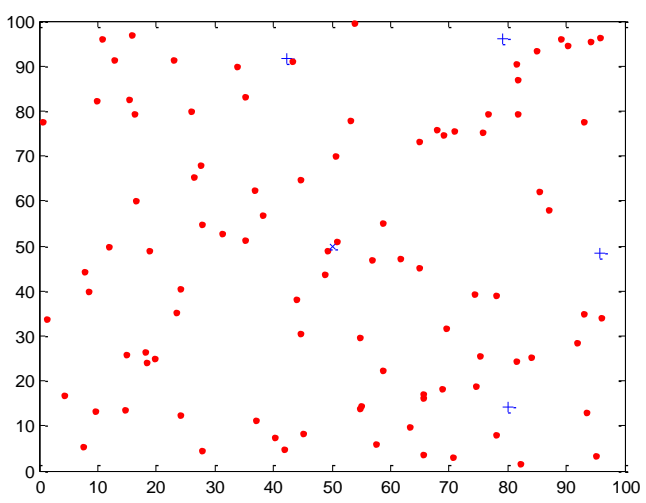

Figure 5: Only advanced nodes are alive 


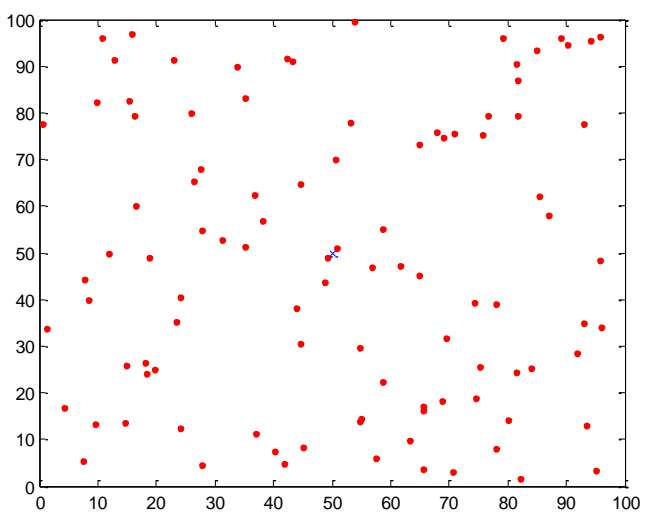

Figure 6: All the nodes are dead

After a certain period of time the first node dies. And gradually the population of normal sensors starts decreasing due to random death of nodes. The transfer of data takes place until the entire advance nodes die and only the sink remains alive as depicted by the above snapshot.

\section{SIMULATION AND OBSERVATION}

50 static wireless sensor nodes are randomly deployed in a $1000 \mathrm{~m} \times 1000 \mathrm{~m}$ area. The communication range of each sensor node is $200 \mathrm{~m}$. Assuming all sensor nodes have radio module for communication and are equipped with an ultrasound transceiver for the distance measurement. It is also assumed that each node has at least 3 one-hop neighbors. According to the design scheme the sensor nodes are randomly deployed connecting each two nodes if the distance between them less than or equal to the communication radius which is shown by the Figure- 6 . Other system parameters are $\mathrm{f}_{\mathrm{c}}=2.5 \mathrm{GHz}, \mathrm{B}=10 \mathrm{kHz}, \alpha=0.4706$, $\mathrm{M}_{\mathrm{l}}=40 \mathrm{~dB}, \mathrm{~N}_{\mathrm{f}}=10 \mathrm{~dB}, \mathrm{G}_{\mathrm{t}} \mathrm{G}_{\mathrm{r}}=5 \mathrm{~dB}, \mathrm{~h}_{\mathrm{t}}=\mathrm{h}_{\mathrm{r}}=1 \mathrm{~m}, \mathrm{~N}_{0} / 2=-174 \mathrm{dBm} / \mathrm{Hz}$, $\mathrm{P}_{\mathrm{ct}}=98.2 \mathrm{~mW}, \mathrm{P}_{\mathrm{cr}}=112.6 \mathrm{~mW}, \mathrm{P}_{\mathrm{b}}=10^{-3}, \mathrm{E}_{\mathrm{DA}}=50 \mathrm{~nJ}, \mathrm{~L}=1000$ bits, $\mathrm{K}_{\mathrm{c}}=8, \mathrm{~L}=1000$. During the communication process, the energy consumed in individual stages can be shown in the following figures.

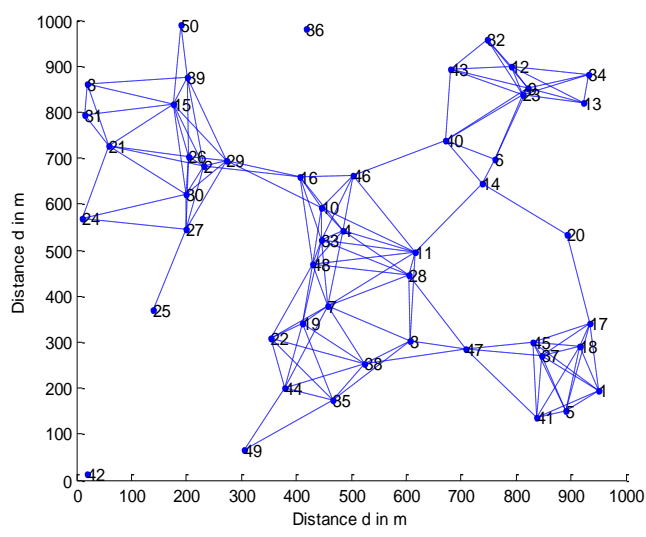

Figure 7: Cluster based Sensor Deployment

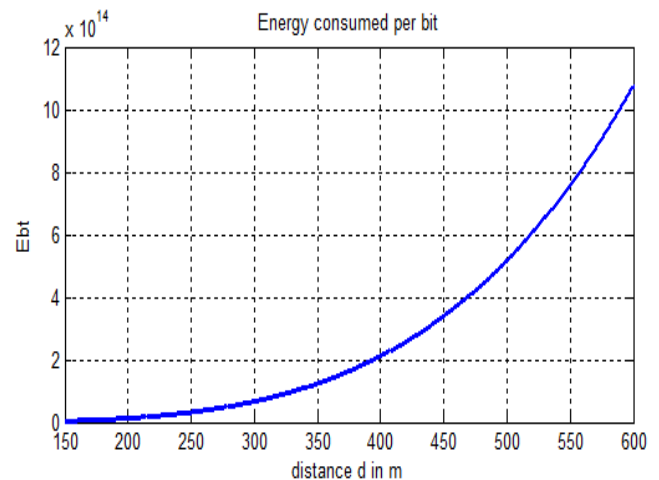

Figure 8: Energy Consumption per bit

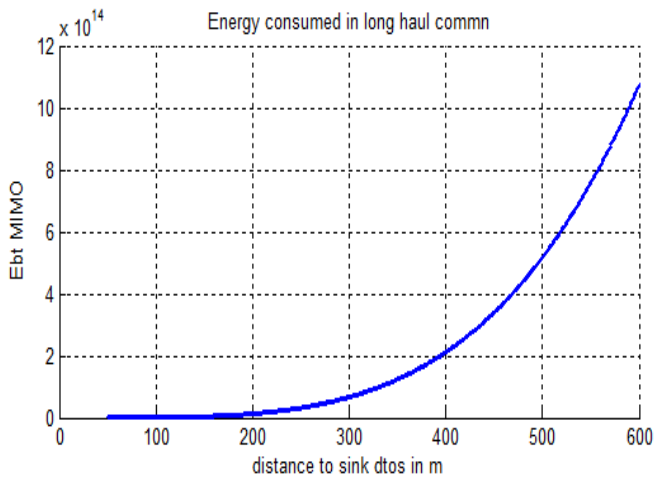

Figure 9: Energy Consumption in long-haul communication

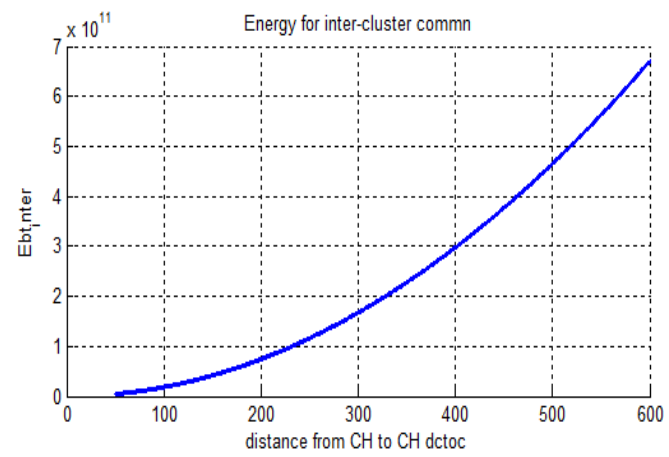

Figure 10: For inter or intra cluster communication

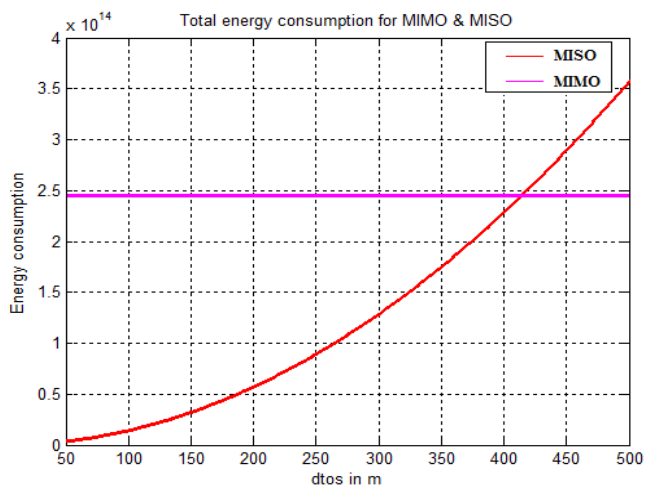

Figure 11: MISO v/s MIMO (4X4) 
In figure 11 , it is clearly shown that although the energy for MIMO is initially high as compared to MISO but after $400 \mathrm{~m}$ of distance the MISO-SCHCT energy continues rising but the proposed MIMO-SCHCT scheme the energy consumption remains constant. If the MIMO-SCHCT size is increased to $8 \mathrm{X} 8$, then also the energy consumption is far below the MIMOLEACH protocol considering the same parameters as shown in figure 12 .

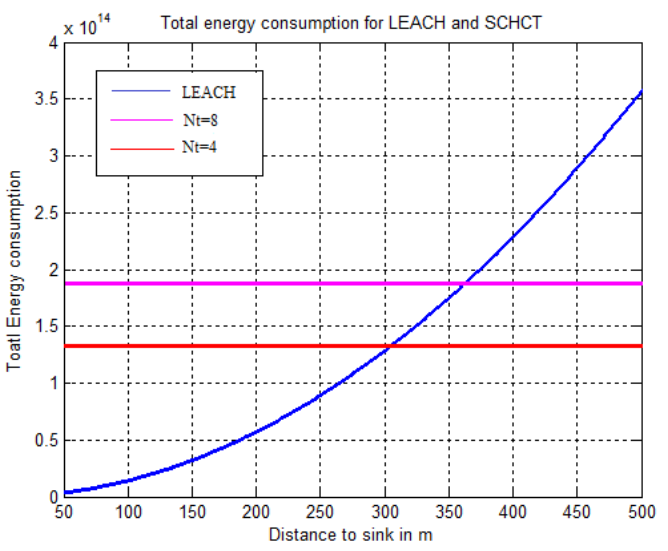

Figure 12: MIMO-SCHCT v/s MIMO- LEACH

Considering 100 sensor nodes in a heterogeneous WSN and 2000 rounds of time, it is found that a 8X8 MIMO with heterogeneous network has more life span than the classic homogenous network as shown in figure 13.

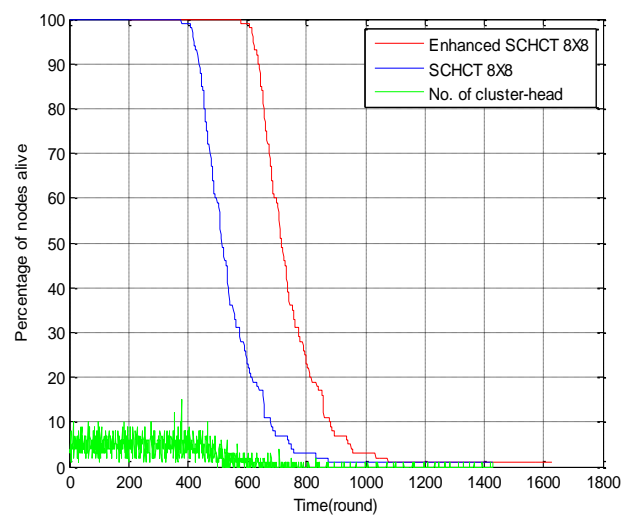

Figure 13: Percentage of nodes alive With MIMO-SCHCT for Homogeneous and Heterogeneous WSN scenario

\section{CONCLUSIONS}

The analysis shows that although the energy consumption in MISO is less in comparison to other traditional techniques but when distance from sink increases MIMO outperforms MISO.A cluster-based cooperative SCHCT-MIMO scheme for multi-hop WSN has been explored to minimize the energy consumption and increase the lifetime of sensor nodes and the performance of the system is highly encouraging. The energy consumption is less when the number of transmitting antenna is minimized. But with the SCHCT scheme, even increasing the MIMO size has also very less impact on energy consumption as compared to MIMO-LEACH protocol. Further deploying a heterogeneous network, we also succeeded in increasing the network lifetime to a large extent.

\section{REFERENCES}

[1] S. Cui, A. J. Goldsmith and A. Bahai, "Energy efficiency of MIMO and Cooperative MIMO Techniques in Sensor Networks," IEEE Journal of Selected Areas in Communications, vol.22, pp.10891098, Aug. 2004.

[2] S. K. Jayaweera, "Virtual MIMO-based Cooperative Communication for Energy-constrained Wireless Sensor Networks," IEEE Trans. On Wireless Communications, vol. 5, pp. 984-989, May 2006.

[3] Y.G. Mei, Y.H. Lu, Y.C. Hu, and C.S. George Lee, "Deployment Strategy for Mobile Robots with Energy and Timing Constraints," Proc. IEEE Int'l Conf. Intelligent Robots and Systems, 2005.

[4] Jie Ding, Danpu Liu, Xin Wang, and Huari Wu. "An energy-efficient virtual MIMO transmission scheme for cluster-based wireless sensor networks", 2010 IEEE 12th International Conference on Communication Technology, 2010.

[5] Yong Yuan, Min Chen and Taekyoung Kwon, "A novel cluster-based cooperative MIMO scheme for multi-hop wireless sensor networks," EURASIP Journal on Wireless Communications and Networking, vol. 2006, pp. 1-9, 2006.

[6] Xiaohua Li, "Energy efficient wireless sensor networks with transmission diversity," IEEE Electronics Letters, vol.39, pp.1753-1755, Nov. 2003.

[7] Y. Yuan, Z. He and M. Chen, "Virtual MIMO- based cross-layer design for wireless sensor networks", IEEE Transactions on Vehicular Technology, vol. 55, no.3, pp. $856-864,2006$.

[8] X. Li, M. Chen and W. Liu, "Application of STBC encoded cooperative transmissions in wireless sensor networks", IEEE Signal Processing Letters, Vol.22, No. 2, pp. 134-1 37, February 2005.

[9] G. Smaragdakis, I. Matta, A. Bestavros, SEP: A Stable Election Protocol for clustered heterogeneous wireless sensor networks, in: Second International Workshop on Sensor and Actor Network Protocols and Applications (SANPA 2004), 2004.

[10] Georgios Smaragdakis, Ibrahim Matta and Azer Bestavros "SEP: A Stable Election Protocol for clustered heterogeneous wireless sensor networks", Boston University. 